\title{
Existence results for Riemann-Liouville fractional neutral evolution equations
}

\author{
Yi-Liang Liu* and Jing-Yun LV
}

\author{
"Correspondence: \\ yiliangliu100@126.com \\ College of Sciences, Guangxi \\ University for Nationalities, Nanning, \\ Guangxi 530006, P.R. China
}

\begin{abstract}
In this paper, by using the fractional power of operators and the theory of measure of noncompactness, we discuss a class of fractional neutral evolution equations with Riemann-Liouville fractional derivative. We establish sufficient conditions for the existence of mild solutions for fractional neutral evolution equations in the cases $C_{0}$ semigroup is compact or noncompact. We give an example to illustrate the applications of the abstract results.
\end{abstract}

Keywords: fractional neutral evolution equations; Riemann-Liouville fractional derivative; mild solutions; analytic semigroup; measure of noncompactness

\section{Introduction}

A strong motivation for studying fractional differential equations comes from the fact that fractional order derivatives and integrals have extensive applications in viscoelasticity, analytical chemistry, electromagnetic, neuron modeling, and biological sciences, and the theory of fractional calculus has attracted great interest from the mathematical science research community. For more details of the theory and applications in this field, see the monographs of Samako et al. [1], Kilbas et al. [2], Miller and Ross [3], Podlubny [4] and the references therein.

Practical problems require definitions of fractional derivatives allowing the utilization of physically interpretable initial conditions. Initial conditions for Caputo fractional derivatives are expressed in terms of initial integer order derivatives. Heymans and Podlubny [5] demonstrated that it is possible to attribute physical meaning to initial conditions expressed in terms of Riemann-Liouville fractional derivatives or integrals on the field of the viscoelasticity, and such initial conditions are more appropriate than physically interpretable initial conditions.

Recently, fractional evolution equations with the Caputo fractional derivative with difference conditions were studied by many authors (see, e.g., [6-15]), but much less is known about the fractional evolution equations with Riemann-Liouville fractional derivative; see [16-18].

In [16], Zhou, Zhang and Shen researched the existence of solutions for a fractional evolution equation with the Riemann-Liouville fractional derivative of the form

$$
\left\{\begin{array}{l}
{ }^{L} D_{0+}^{q} x(t)=A x(t)+(F x)(t), \quad t \in J^{\prime}:=(0, a], 0<q<1, \\
\left(I_{0^{+}}^{1-q} x\right)(0)+g(x)=x_{0},
\end{array}\right.
$$

@2014 Liu and Lv; licensee Springer. This is an Open Access article distributed under the terms of the Creative Commons Attribution License (http://creativecommons.org/licenses/by/2.0), which permits unrestricted use, distribution, and reproduction in any medium, provided the original work is properly cited. 
where ${ }^{L} D_{0+}^{\alpha}$ is the Riemann-Liouville fractional derivative of order $q, I_{0^{+}}^{1-q}$ is the RiemannLiouville integral of order $1-q, A$ is the infinitesimal generator of a $C_{0}$-semigroup $\{T(t), t \geq 0\}$ on a Banach space $X . F: C\left(J^{\prime}, X\right) \rightarrow L\left(J^{\prime}, X\right)$ and $g: C\left(J^{\prime}, X\right) \rightarrow L\left(J^{\prime}, X\right)$ are given functions satisfying some assumptions.

However, fractional neutral evolution equations with a Riemann-Liouville fractional derivative have not been considered in the literature. Motivated by the above work, the aim of this paper is to study the existence of solutions for fractional neutral evolution equations with a Riemann-Liouville fractional derivative of the form

$$
\left\{\begin{array}{l}
{ }^{L} D_{0+}^{q}[x(t)-h(t, x(t))]=-A x(t)+f(t, x(t)), \quad t \in J^{\prime}=(0, b], 0<q<1, \\
\left.I_{0^{+}}^{1-q}[x(t)-h(t, x(t))]\right|_{t=0}=x_{0} \in X,
\end{array}\right.
$$

where ${ }^{L} D_{0+}^{q}$ is the Riemann-Liouville fractional derivative of order $q$ with the lower limit zero, $I_{0^{+}}^{1-q}$ is the Riemann-Liouville integral of order $1-q$. $X$ is a Banach space, $-A: D(A) \subseteq$ $X \rightarrow X$ is the infinitesimal generator of an analytic semigroup $\{T(t), t \geq 0\}$ on a Banach space $X . h$ and $f$ are given functions satisfying some assumptions.

Neutral differential equations arise in many areas of applied mathematics and for this reason these equations have received much attention in the last few decades. The literature related to fractional neutral differential equations is very extensive; see for instance [12$14,19]$. The results we obtained in this paper are a generalization and continuation of the recent results on this issue.

The rest of this paper is organized as follows. In Section 2, some notations and preparation results are given. In Section 3, by using the fractional power of operators and the technique of using a measure of noncompactness, we give existence results for problem (1) under both compactness and noncompactness conditions on the $C_{0}$ semigroup. We present an example to demonstrate our main results in Section 4. Finally, the manuscript ends with our conclusions.

\section{Preliminaries}

In this section, we introduce the notations, definitions, and preliminary facts that will be used in the remainder of this paper.

In this paper, we assume that $X$ is a Banach space. Let $C(J, X)$ denote the Banach space of all $X$-value continuous functions from $J=[0, b]$ into $X$ with the norm $\|x\|_{C(J, X)}=$ $\sup _{t \in J}\|x(t)\|_{X}$. For measurable functions $m: J \rightarrow \mathbb{R}$, we define the norm $\|m\|_{L^{p}(J, \mathbb{R})}:=$ $\left(\int_{J}|m(t)|^{p} d t\right)^{\frac{1}{p}}, 1 \leq p<\infty . L^{p}(J, \mathbb{R})(1 \leq p<\infty)$ is the Banach space of all Lebesgue measurable functions from $J$ into $\mathbb{R}$ with $\|m\|_{L^{p}(, \mathbb{R})}<\infty$. Let $L^{p}(J, X)$ denote the Banach space of functions $m: J \rightarrow X$ which are Bochner integrable normed by $\|m\|_{L^{p}(J, X)}$. Let $J^{\prime}=(0, b]$, to define the mild solution of (1), we also consider the Banach space $C_{1-q}(J, X)=\{x \in$ $\left.C\left(J^{\prime}, X\right): t^{1-q} x(t) \in C(J, X)\right\}$ with the norm $\|x\|_{C_{1-q}}=\sup _{t \in J}\left\{t^{1-q}\|x(t)\|_{X}\right\}$. It is easy to see $\left(C_{1-q}(J, X),\|\cdot\|_{C_{1-q}}\right)$ is a Banach space.

First, let us recall the following definitions and properties of the fractional calculus. For more details, one can refer to [2-4].

Definition 2.1 The fractional integral of order $q$ with the lower limit zero for a function $f$ is defined as

$$
I_{0^{+}}^{q} f(t)=\frac{1}{\Gamma(q)} \int_{0}^{t} \frac{f(s)}{(t-s)^{1-q}} d s, \quad t>0, q>0
$$


provided the right side is point-wise defined on $[0, \infty)$, where $\Gamma(\cdot)$ is the gamma function.

Definition 2.2 The Riemann-Liouville derivative of order $q$ with the lower limit zero for a function $f:[0, \infty) \rightarrow \mathbb{R}$ can be written as

$$
{ }^{L} D_{0+}^{q} f(t)=\frac{1}{\Gamma(n-q)}\left(\frac{d}{d t}\right)^{n} \int_{0}^{t} \frac{f(s)}{(t-s)^{q-n+1}} d s, \quad t>0, n-1<q<n .
$$

Definition 2.3 The Caputo derivative of order $q$ for a function $f:[0, \infty) \rightarrow \mathbb{R}$ can be written as

$$
{ }^{c} D_{0^{+}}^{q} f(t)={ }^{L} D^{q}\left[f(t)-\sum_{k=0}^{n-1} \frac{t^{k}}{k !} f^{(k)}(0)\right], \quad t>0, n-1<q<n .
$$

\section{Remark 2.4}

(i) If $f(t) \in C^{n}[0, \infty)$, then

$$
{ }^{c} D_{0^{+}}^{q} f(t)=\frac{1}{\Gamma(n-q)} \int_{0}^{t} \frac{f^{(n)}(s)}{(t-s)^{q+1-n}} d s=I_{0^{+}}^{n-q} f^{(n)}(t), \quad t>0, n-1<q<n .
$$

(ii) The Caputo derivative of a constant is equal to zero.

(iii) If $f$ is an abstract function with values in $X$, then integrals which appear in Definitions 2.1 and 2.2 are taken in Bochner's sense.

Throughout this paper, let $-A$ be the infinitesimal generator of an analytic semigroup $\{T(t)\}_{t \geq 0}$ of uniformly bounded linear operators on $X$; this means that there exists $M \geq 1$, such that $M:=\sup _{t \in[0, \infty)}\|T(t)\|<\infty$. Let $0 \in \rho(A)$, where $\rho(A)$ is the resolvent set of $A$. Then for $0<\eta \leq 1$, it is possible to define the fractional power $A^{\eta}$ as a closed linear operator on its domain $D\left(A^{\eta}\right)$. Moreover, the subspace $D\left(A^{\eta}\right)$ is dense in $X$ and the expression

$$
\|x\|_{\eta}=\left\|A^{\eta} x\right\|, \quad x \in D\left(A^{\eta}\right),
$$

defines a norm on $D\left(A^{\eta}\right)$. We denote by $X_{\eta}$ the Banach space $D\left(A^{\eta}\right)$ normed with $\|x\|_{\eta}$.

For analytic semigroup $\{T(t)\}_{t \geq 0}$, the following property will be used:

(i) for any $\eta \in(0,1]$, there exists a positive constant $C_{\eta}$ such that

$$
\left\|A^{\eta} T(t)\right\| \leq \frac{C_{\eta}}{t^{\eta}}, \quad 0<t \leq b .
$$

For more details as regards the above preliminaries, we refer the reader to [20, 21].

Next, we recall some definitions and properties of measure of noncompactness. For more details, we refer the reader to $[22,23]$ and the references therein.

Definition 2.5 Let $X$ be a Banach space, $\mathcal{P}(X)$ denote the collection of all nonempty subsets of $X$, and $(\mathcal{A}, \geq)$ a partially ordered set. A map $\beta: \mathcal{P}(X) \rightarrow \mathcal{A}$ is called a measure of noncompactness on $X$, MNC for short, if

$$
\beta(\overline{c o} \Omega)=\beta(\Omega)
$$

for every $\Omega \in \mathcal{P}(X)$, where $\overline{c o} \Omega$ is the closure of convex hull of $\Omega$. 
Definition 2.6 A measure of noncompactness $\beta$ is called

(a) monotone if $\Omega_{0}, \Omega_{1} \in \mathcal{P}(X), \Omega_{0} \subseteq \Omega_{1}$ implies $\beta\left(\Omega_{0}\right) \leq \beta\left(\Omega_{1}\right)$;

(b) nonsingular if $\beta(\{a\} \cup \Omega)=\beta(\Omega)$ for every $a \in X, \Omega \in \mathcal{P}(X)$;

(c) invariant with respect to the union with compact sets if $\beta(K \cup \Omega)=\beta(\Omega)$ for every relatively compact set $K \subseteq X$ and $\Omega \in \mathcal{P}(X)$;

(d) semi-additive if $\beta\left(\Omega_{0} \cup \Omega_{1}\right)=\max \left(\beta\left(\Omega_{0}\right), \beta\left(\Omega_{1}\right)\right)$ for every $\Omega_{0}, \Omega_{1} \in \mathcal{P}(X)$;

(e) regular if the condition $\beta(\Omega)=0$ is equivalent to the relative compactness of $\Omega$.

One of the most important examples of the measure of noncompactness is the Kuratowski measure of noncompactness $\alpha$ defined on each bounded subset $B$ of $X$ by

$\alpha(B)=\inf \{\varepsilon>0: B$ is covered by a finite number of sets with diameter $\leq \varepsilon\}$.

It is well known that the Kuratowski measure of noncompactness $\alpha$ enjoys the above properties (a)-(e) and other properties (see [24-26]). We have

(f) $\alpha\left(B_{1}+B_{2}\right) \leq \alpha\left(B_{1}\right)+\alpha\left(B_{2}\right)$, where $B_{1}+B_{2}=\left\{x+y: x \in B_{1}, y \in B_{2}\right\}$;

(g) $\alpha(\lambda B) \leq|\lambda| \alpha(B)$ for any $\lambda \in R$.

For any $W \subset C(J, X)$, we define

$$
\int_{0}^{t} W(s) d s=\left\{\int_{0}^{t} u(s) d s: u \in W\right\}, \quad \text { for } t \in J=[0, b]
$$

where $W(s)=\{u(s) \in X: u \in W\}$.

Proposition 2.7 If $W \subset C(J, X)$ is bounded and equicontinuous, then $\overline{c o} W \subset C(J, X)$ is also bounded and equicontinuous.

Proposition 2.8 [27] If $W \subset C(J, X)$ is bounded and equicontinuous, then $t \mapsto \alpha(W(t))$ is continuous on $J$ and

$$
\alpha(W)=\max _{t \in J} \alpha(W(t)), \quad \alpha\left(\int_{0}^{t} W(s) d s\right) \leq \int_{0}^{t} \alpha(W(s)) d s, \quad \text { for } t \in[0, b] .
$$

Proposition 2.9 [28] $X$ is a Banach space, if $B \subset X$ is bounded, then for each $\varepsilon>0$, there is a sequence $\left\{u_{n}\right\}_{n=1}^{\infty} \subset B$, such that

$$
\alpha(B) \leq 2 \alpha\left(\left\{u_{n}\right\}_{n=1}^{\infty}\right)+\varepsilon
$$

Proposition 2.10 [29] Let $\left\{u_{n}\right\}_{n=1}^{\infty}$ be a sequence of Bochner integrable functions from $J$ into $X$ with $\left\|u_{n}(t)\right\| \leq m(t)$ for almost all $t \in J$ and every $n \geq 1$, where $m \in L\left(J, R^{+}\right)$, then the function $\varphi(t)=\alpha\left(\left\{u_{n}(t)\right\}_{n=1}^{\infty}\right)$ belongs to $L\left(J, R^{+}\right)$and satisfies

$$
\alpha\left(\int_{0}^{t} u_{n}(s) d s: n \geq 1\right) \leq 2 \int_{0}^{t} \varphi(s) d s
$$

Lemma 2.11 Let $B$ be a closed and convex subset of a Banach space X. Suppose $F: B \rightarrow B$ is a continuous operator and $F(B)$ is bounded. For each bounded subset $B_{0} \subset B$, set

$$
F^{1}\left(B_{0}\right)=F\left(B_{0}\right), \quad F^{n}\left(B_{0}\right)=F\left(\overline{c o}\left(F^{n-1}\left(B_{0}\right)\right)\right), \quad n=2,3, \ldots
$$


If there exist a constant $0 \leq k<1$ and a positive integer $n_{0}$ such that, for any bounded subset $B_{0} \subset B$,

$$
\alpha\left(F^{n_{0}}\left(B_{0}\right)\right) \leq k \alpha\left(B_{0}\right)
$$

then there exists a nonempty, compact, and convex subset $\widehat{D} \subset B$ such that $F: \widehat{D} \rightarrow \widehat{D}$ and $\alpha(F(\widehat{D}))=0$.

According to Lemma 2.4 in [30], we can obtain the results immediately.

The map $F: B \subseteq X \rightarrow X$ is said to be an $\alpha$-contraction if there exists a positive constant $k<1$ such that $\alpha\left(F\left(B_{0}\right)\right) \leq k \alpha\left(B_{0}\right)$ for any bounded closed subset $B_{0} \subseteq B$.

Lemma 2.12 (Darbo-Sadovskii's fixed point theorem) [24] If B is a bounded closed convex subset of a Banach space $X$, the continuous map $F: B \rightarrow B$ is an $\alpha$-contraction, then the map $F$ has at least one fixed point in $B$.

On the basis of $[12,16]$, we give the following definition of a mild solution of system (1).

Definition 2.13 By a mild solution of system (1), we mean the function $x \in C_{1-q}(J, X)$ which satisfies

$$
\begin{aligned}
x(t)= & t^{q-1} T_{q}(t) x_{0}+h(t, x(t))+\int_{0}^{t}(t-s)^{q-1} A T_{q}(t-s) h(s, x(s)) d s \\
& +\int_{0}^{t}(t-s)^{q-1} T_{q}(t-s) f(s, x(s)) d s, \quad t \in(0, b],
\end{aligned}
$$

where

$$
T_{q}(t)=q \int_{0}^{\infty} \theta M_{q}(\theta) T\left(t^{q} \theta\right) d \theta
$$

and $M_{q}$ is a probability density function which is defined by

$$
M_{q}(\theta)=\sum_{n=1}^{\infty} \frac{(-\theta)^{n-1}}{(n-1) ! \Gamma(1-q n)}, \quad 0<q<1, \theta \in \mathbb{C} .
$$

Lemma 2.14 [12] The operator $T_{q}(t)$ has the following properties.

(i) For any fixed $t \geq 0, T_{q}(t)$ is linear and bounded operators, i.e., for any $x \in X$,

$$
\left\|T_{q}(t) x\right\| \leq \frac{M}{\Gamma(q)}\|x\|
$$

(ii) $T_{q}(t)(t \geq 0)$ is strongly continuous, which means that, for $\forall x \in X$ and $0<t^{\prime}<t^{\prime \prime} \leq b$, we have

$$
\left\|T_{q}\left(t^{\prime \prime}\right) x-T_{q}\left(t^{\prime}\right) x\right\| \rightarrow 0 \quad \text { as } t^{\prime \prime} \rightarrow t^{\prime}
$$

(iii) for every $t>0, T_{q}(t)$ is a compact operator if $T(t)$ is also compact. 
Lemma 2.15 [12] For any $\beta \in(0,1), x \in D\left(A^{\beta}\right)$ and $\eta \in(0,1]$, we have

$$
A T_{q}(t) x=A^{1-\beta} T_{q}(t) A^{\beta} x, \quad 0 \leq t \leq b,
$$

and

$$
\left\|A^{\eta} T_{q}(t)\right\| \leq \frac{q C_{\eta} \Gamma(2-\eta)}{t^{q \eta} \Gamma(1+q(1-\eta))}, \quad 0<t \leq b .
$$

\section{Existence of mild solutions}

Before stating and proving the main results, we introduce the following assumptions.

$\left(\mathrm{H}_{1}\right) T(t)$ is a compact operator for every $t>0$.

$\left(\mathrm{H}_{2}\right)$ For almost all $t \in J$, the function $f(t, \cdot): X \rightarrow X$ is continuous and for each $z \in X$, the function $f(\cdot, z): J \rightarrow X$ is strongly measurable.

$\left(\mathrm{H}_{3}\right)$ There exist a function $\phi(t) \in L^{\frac{1}{p}}\left(J, \mathbb{R}^{+}\right), p \in(0, q)$, and a constant $c>0$ such that

$$
\|f(t, x(t))\| \leq \phi(t)+c t^{1-q}\|x(t)\|, \quad \forall x \in C_{1-q}(J, X), t \in J,
$$

and the operator $G: \Phi \rightarrow L^{\frac{1}{P}}(J, X)$ is continuous, where

$$
(G x)(t)=f(t, x(t)), \quad \forall x \in \Phi, \Phi \text { is any bounded set of } C_{1-q}(J, X) .
$$

$\left(\mathrm{H}_{4}\right) h: J^{\prime} \times X \rightarrow X$ is a continuous function and there exist a constant $\beta \in(0,1)$ and $L>0$ such that $h \in D\left(A^{\beta}\right)$ and for any $z, y \in X, t \in J^{\prime}$, the function $A^{\beta} h(\cdot, z)$ is strongly measurable and $A^{\beta} h$ satisfies

$$
\left\|A^{\beta} h(t, x(t))-A^{\beta} h(t, y(t))\right\| \leq L t^{1-q}\|x(t)-y(t)\|, \quad \text { for all } x, y \in C_{1-q}(J, X), t \in J^{\prime},
$$

and the inequality

$$
\left\|A^{\beta} h(t, x(t))\right\| \leq L\left(1+\|x\|_{C_{1-q}}\right), \quad \forall t \in J^{\prime}, x \in C_{1-q}(J, X) .
$$

For brevity, let

$$
\begin{aligned}
& M_{0}=\left\|A^{-\beta}\right\|, \\
& M_{1}=b^{1-q} M_{0} L+\frac{C_{1-\beta} \Gamma(1+\beta) L b^{1-q+q \beta}}{\beta \Gamma(1+q \beta)}+\frac{M c b}{\Gamma(1+q)}, \\
& M_{2}=\frac{M}{\Gamma(q)}\left\|x_{0}\right\|+b^{1-q} M_{0} L+\frac{C_{1-\beta} \Gamma(1+\beta) L b^{1-q+q \beta}}{\beta \Gamma(1+q \beta)}+\frac{M}{\Gamma(q)}\left(\frac{1-p}{q-p} b\right)^{1-p}\|\phi\|_{L^{\frac{1}{p}}(J, X)} .
\end{aligned}
$$

Define an operator $F$ on $C_{1-q}(J, X)$ by

$$
\begin{aligned}
(F x)(t)= & t^{q-1} T_{q}(t) x_{0}+h(t, x(t))+\int_{0}^{t}(t-s)^{q-1} A T_{q}(t-s) h(s, x(s)) d s \\
& +\int_{0}^{t}(t-s)^{q-1} T_{q}(t-s) f(s, x(s)) d s, \quad t \in(0, b] .
\end{aligned}
$$


For any $x \in C_{1-q}(J, X)$, let

$$
(F x)(t)=\left(F_{1} x\right)(t)+\left(F_{2} x\right)(t)
$$

where

$$
\begin{aligned}
& \left(F_{1} x\right)(t)=t^{q-1} T_{q}(t) x_{0}+\int_{0}^{t}(t-s)^{q-1} T_{q}(t-s) f(s, x(s)) d s, \quad t \in(0, b], \\
& \left(F_{2} x\right)(t)=h(t, x(t))+\int_{0}^{t}(t-s)^{q-1} A T_{q}(t-s) h(s, x(s)) d s, \quad t \in(0, b] .
\end{aligned}
$$

Assume that $M_{1}<1$, and let

$$
B_{r}=\left\{x \in C_{1-q}(J, X):\|x\|_{C_{1-q}} \leq r\right\}, \quad \text { where } r \geq \frac{M_{2}}{1-M_{1}} .
$$

Lemma 3.1 If the assumptions $\left(\mathrm{H}_{1}\right)-\left(\mathrm{H}_{3}\right)$ are satisfied and $M_{1}<1$, then $F_{1}\left(B_{r}\right)$ is relatively compact set in $C_{1-q}(J, X)$.

Proof Using $\left(\mathrm{H}_{1}\right)-\left(\mathrm{H}_{3}\right)$ and Lemma 2.14 , one can easily prove that $F_{1} x \in C_{1-q}(J, X)$ for any $x \in C_{1-q}(J, X)$. Then $F_{1}$ is well defined on $C_{1-q}(J, X)$. Let

$$
\Omega=\left\{y \in C(J, X): y(t)=t^{1-q}\left(F_{1} x\right)(t), x \in B_{r}\right\} .
$$

For the sake of convenience, we divide the proof into several steps.

Step 1: For any $y \in \Omega,\|y\|_{C(J, X)} \leq r$.

For any $x \in B_{r}$ and $t \in J$, taking into account the imposed assumptions, applying Lemma 2.14 and the Hölder inequality, we obtain

$$
\begin{aligned}
t^{1-q}\left\|\left(F_{1} x\right)(t)\right\| & \leq\left\|T_{q}(t) x_{0}\right\|+t^{1-q}\left\|\int_{0}^{t}(t-s)^{q-1} T_{q}(t-s) f(s, x(s)) d s\right\| \\
& \leq \frac{M}{\Gamma(q)}\left\|x_{0}\right\|+\frac{M t^{1-q}}{\Gamma(q)} \int_{0}^{t}(t-s)^{q-1}\left[\phi(s)+c s^{1-q}\|x(s)\|\right] d s \\
& \leq \frac{M}{\Gamma(q)}\left\|x_{0}\right\|+\frac{M}{\Gamma(q)}\left(\frac{1-p}{q-p} b\right)^{1-p}\|\phi\|_{L^{\frac{1}{p}}(J, X)}+\frac{M c b r}{\Gamma(q+1)} \\
& \leq r .
\end{aligned}
$$

Hence, for any $y \in \Omega,\|y\|_{C(J, X)} \leq r$.

Step 2: $\Omega \subseteq C(J, X)$ is equicontinuous.

For any $y \in \Omega$, let $0=t_{1}<t_{2} \leq b$. Taking $\left(\mathrm{H}_{3}\right)$ into account, we get

$$
\begin{aligned}
& \left\|y\left(t_{2}\right)-y(0)\right\| \\
& \quad \leq\left\|T_{q}\left(t_{2}\right) x_{0}-T_{q}(0) x_{0}\right\|+\left\|t_{2}^{1-q} \int_{0}^{t_{2}}\left(t_{2}-s\right)^{q-1} T_{q}\left(t_{2}-s\right) f(s, x(s)) d s\right\| \\
& \quad \leq\left\|T_{q}\left(t_{2}\right) x_{0}-T_{q}(0) x_{0}\right\|+\frac{M}{\Gamma(q)}\left(\frac{1-p}{q-p} t_{2}\right)^{1-p}\|\phi\|_{L^{\frac{1}{p}}(J, X)}+\frac{M c r t_{2}}{\Gamma(q+1)} \\
& \quad \rightarrow 0, \quad \text { as } t_{2} \rightarrow 0^{+} .
\end{aligned}
$$


For $0<t_{1}<t_{2} \leq b$, selecting $\varepsilon>0$ sufficiently small. Taking $\left(\mathrm{H}_{3}\right)$ and $\left(\mathrm{H}_{4}\right)$ into account, we have

$$
\begin{aligned}
\| y\left(t_{2}\right) & -y\left(t_{1}\right) \| \\
\leq & \left\|T_{q}\left(t_{2}\right) x_{0}-T_{q}\left(t_{1}\right) x_{0}\right\|+\left\|t_{2}^{1-q} \int_{t_{1}}^{t_{2}}\left(t_{2}-s\right)^{q-1} T_{q}\left(t_{2}-s\right) f(s, x(s)) d s\right\| \\
& +\left\|\int_{0}^{t_{1}}\left[t_{2}^{1-q}\left(t_{2}-s\right)^{q-1}-t_{1}^{1-q}\left(t_{1}-s\right)^{q-1}\right] T_{q}\left(t_{2}-s\right) f(s, x(s)) d s\right\| \\
& +\left\|t_{1}^{1-q} \int_{0}^{t_{1}}\left(t_{1}-s\right)^{q-1}\left[T_{q}\left(t_{2}-s\right)-T_{q}\left(t_{1}-s\right)\right] f(s, x(s)) d s\right\| \\
\leq & \left\|T_{q}\left(t_{2}\right) x_{0}-T_{q}\left(t_{1}\right) x_{0}\right\|+\frac{M b^{1-q}}{\Gamma(q)} \int_{t_{1}}^{t_{2}}\left(t_{2}-s\right)^{q-1}[\phi(s)+c r] d s \\
& +\frac{M}{\Gamma(q)} \int_{0}^{t_{1}}\left[t_{1}^{1-q}\left(t_{1}-s\right)^{q-1}-t_{2}^{1-q}\left(t_{2}-s\right)^{q-1}\right](\phi(s)+c r) d s \\
& +\left\|\int_{0}^{t_{1}-\varepsilon} t_{1}^{1-q}\left(t_{1}-s\right)^{q-1}\left[T_{q}\left(t_{2}-s\right)-T_{q}\left(t_{1}-s\right)\right] f(s, x(s)) d s\right\| \\
& +\left\|\int_{t_{1}-\varepsilon}^{t_{1}} t_{1}^{1-q}\left(t_{1}-s\right)^{q-1}\left[T_{q}\left(t_{2}-s\right)-T_{q}\left(t_{1}-s\right)\right] f(s, x(s)) d s\right\| \\
\leq & I_{1}+I_{2}+I_{3}+I_{4}+I_{5},
\end{aligned}
$$

where

$$
\begin{aligned}
& I_{1}=\left\|T_{q}\left(t_{2}\right) x_{0}-T_{q}\left(t_{1}\right) x_{0}\right\|, \\
& I_{2}=\frac{M b^{1-q}}{\Gamma(q)} \int_{t_{1}}^{t_{2}}\left(t_{2}-s\right)^{q-1}[\phi(s)+c r] d s, \\
& I_{3}=\frac{M}{\Gamma(q)} \int_{0}^{t_{1}}\left[t_{1}^{1-q}\left(t_{1}-s\right)^{q-1}-t_{2}^{1-q}\left(t_{2}-s\right)^{q-1}\right](\phi(s)+c r) d s, \\
& I_{4}=\sup _{s \in\left[0, t_{1}-\varepsilon\right]}\left\|T_{q}\left(t_{2}-s\right)-T_{q}\left(t_{1}-s\right)\right\|\left[\left(\frac{1-p}{q-p} b\right)^{1-p}\|\phi\|_{L^{\frac{1}{p}}(J, X)}+\frac{b c r}{q}\right], \\
& I_{5}=\frac{2 M b^{1-q}}{\Gamma(q)} \int_{t_{1}-\varepsilon}^{t_{1}}\left(t_{1}-s\right)^{q-1}[\phi(s)+c r] d s .
\end{aligned}
$$

According to Lemma 2.14(ii), it is easy to see that $I_{1} \rightarrow 0$ as $t_{2} \rightarrow t_{1}$. Applying the absolute continuity of the Lebesgue integral, we see that $I_{2}$ and $I_{5}$ tend to zero independently of $x \in B_{r}$ as $t_{2} \rightarrow t_{1}, \varepsilon \rightarrow 0$. Noting that

$$
0<\left[t_{1}^{1-q}\left(t_{1}-s\right)^{q-1}-t_{2}^{1-q}\left(t_{2}-s\right)^{q-1}\right](\phi(s)+c r)<t_{1}^{1-q}\left(t_{1}-s\right)^{q-1}(\phi(s)+c r),
$$

and that $\int_{0}^{t_{1}} t_{1}^{1-q}\left(t_{1}-s\right)^{q-1}(\phi(s)+c r) d s$ exists, by the Lebesgue dominated convergence theorem, we see that $I_{3}$ tends to zero independently of $x \in B_{r}$ as $t_{2} \rightarrow t_{1}$. Since $\left(\mathrm{H}_{1}\right)$ and Lemma 2.14 imply the continuity of $T_{q}(t)(t>0)$ in $t$ in the uniform operator topology, it is easy to see that $I_{4}$ tends to zero independently of $x \in B_{r}$ as $t_{2} \rightarrow t_{1}$. Thus, $\left\|y\left(t_{2}\right)-y\left(t_{1}\right)\right\|$ tends to zero independently of $x \in B_{r}$ as $t_{2} \rightarrow t_{1}$, which means that $\Omega$ is equicontinuous. 
Step 3: For any $t \in[0, b], \Omega(t)=\{y(t), y \in \Omega\}$ is relatively compact in $X$.

This is trivial for $t=0$, since $\Omega(0)=\left\{\frac{x_{0}}{\Gamma(q)}\right\}$. So it is only necessary to consider $0<t \leq b$. Let $0<t \leq b$ be fixed. For $\forall \varepsilon \in(0, t), \forall \delta>0$, define

$$
\begin{aligned}
y^{\varepsilon, \delta}(t)= & q \int_{\delta}^{\infty} \theta M_{q}(\theta) T\left(t^{q} \theta\right) x_{0} d \theta \\
& +q t^{1-q} \int_{0}^{t-\varepsilon} \int_{\delta}^{\infty}(t-s)^{q-1} \theta M_{q}(\theta) T\left((t-s)^{q} \theta\right) f(s, x(s)) d \theta d s \\
\leq & T\left(\varepsilon^{q} \delta\right)\left\{q \int_{\delta}^{\infty} \theta M_{q}(\theta) T\left(t^{q} \theta-\varepsilon^{q} \delta\right) x_{0} d \theta\right. \\
& \left.+q t^{1-q} \int_{0}^{t-\varepsilon} \int_{\delta}^{\infty}(t-s)^{q-1} \theta M_{q}(\theta) T\left((t-s)^{q} \theta-\varepsilon^{q} \delta\right) f(s, x(s)) d \theta d s\right\} .
\end{aligned}
$$

Then from the compactness of $T\left(\varepsilon^{q} \delta\right)\left(\varepsilon^{q} \delta>0\right)$, we find that the set $\Omega^{\varepsilon, \delta}(t)=\left\{y^{\varepsilon, \delta}(t), y \in \Omega\right\}$ is relatively compact in $X$ for $\forall \varepsilon \in(0, t)$ and $\forall \delta>0$.

Moreover, we have

$$
\begin{aligned}
\| y(t) & -y^{\varepsilon, \delta}(t) \| \\
\leq & \left\|q \int_{0}^{\delta} \theta M_{q}(\theta) T\left(t^{q} \theta\right) x_{0} d \theta\right\| \\
& +\left\|q t^{1-q} \int_{0}^{t} \int_{0}^{\delta}(t-s)^{q-1} \theta M_{q}(\theta) T\left((t-s)^{q} \theta\right) f(s, x(s)) d \theta d s\right\| \\
& +\left\|q t^{1-q} \int_{t-\varepsilon}^{t} \int_{\delta}^{\infty}(t-s)^{q-1} \theta M_{q}(\theta) T\left((t-s)^{q} \theta\right) f(s, x(s)) d \theta d s\right\| \\
\leq & q M\left\|x_{0}\right\| \int_{0}^{\delta} \theta M_{q}(\theta) d \theta+q M b^{1-q} \int_{0}^{t}(t-s)^{q-1}[\phi(s)+c r] d s \int_{0}^{\delta} \theta M_{q}(\theta) d \theta \\
& +q M b^{1-q} \int_{t-\varepsilon}^{t}(t-s)^{q-1}[\phi(s)+c r] d s \int_{0}^{\infty} \theta M_{q}(\theta) d \theta \\
\leq & q M\left\|x_{0}\right\| \int_{0}^{\delta} \theta M_{q}(\theta) d \theta+q M b^{1-q} \int_{0}^{t}(t-s)^{q-1}[\phi(s)+c r] d s \int_{0}^{\delta} \theta M_{q}(\theta) d \theta \\
& +\frac{M b^{1-q}}{\Gamma(q)} \int_{t-\varepsilon}^{t}(t-s)^{q-1}[\phi(s)+c r] d s \rightarrow 0, \quad \text { as } \varepsilon \rightarrow 0, \delta \rightarrow 0 .
\end{aligned}
$$

Therefore, there are relatively compact sets arbitrarily close to the set $\Omega(t), t>0$. Hence the set $\Omega(t), t>0$ is also relatively compact in $X$.

As a consequence of Step 1-Step 3, with the Arzola-Ascoli theorem, we can conclude that $\Omega \subseteq C(J, X)$ is a relatively compact set. Keeping in mind the relationship of $\Omega$ and $F_{1}\left(B_{r}\right)$, one can easily prove that $F_{1}\left(B_{r}\right)$ is a relatively compact set in $C_{1-q}(J, X)$. The proof is complete.

\subsection{The case that $T(t)$ is compact}

Theorem 3.2 Assume that hypotheses $\left(\mathrm{H}_{1}\right)-\left(\mathrm{H}_{4}\right)$ hold and $M_{1}<1$, then system (1) has at least one mild solution. 
Proof Using $\left(\mathrm{H}_{1}\right)-\left(\mathrm{H}_{4}\right)$, Lemma 2.14 and Lemma 2.15 , one can easily prove that $F x \in$ $C_{1-q}(J, X)$ for any $x \in C_{1-q}(J, X)$. Then $F$ is well defined on $C_{1-q}(J, X)$. We will show that $F$ satisfies all conditions of Lemma 2.12. The proof will be given in several steps.

For any $x \in B_{r}$ and $t \in J$, taking into account the imposed assumptions, applying Lemma 2.14 and the Hölder inequality, we obtain

$$
\begin{aligned}
t^{1-q}\|(F x)(t)\| \leq & \left\|T_{q}(t) x_{0}\right\|+\left\|t^{1-q} h(t, x(t))\right\| \\
& +\left\|t^{1-q} \int_{0}^{t}(t-s)^{q-1} A T_{q}(t-s) h(s, x(s)) d s\right\| \\
& +\left\|t^{1-q} \int_{0}^{t}(t-s)^{q-1} T_{q}(t-s) f(s, x(s)) d s\right\| \\
\leq & \frac{M}{\Gamma(q)}\left\|x_{0}\right\|+\left\|t^{1-q} A^{-\beta} A^{\beta} h(t, x(t))\right\| \\
& +\left\|t^{1-q} \int_{0}^{t}(t-s)^{q-1} A^{1-\beta} T_{q}(t-s) A^{\beta} h(s, x(s)) d s\right\| \\
& +\frac{M t^{1-q}}{\Gamma(q)} \int_{0}^{t}(t-s)^{q-1}\left[\phi(s)+c s^{1-q}\|x(s)\|\right] d s \\
\leq & \frac{M}{\Gamma(q)}\left\|x_{0}\right\|+b^{1-q} M_{0} L(1+r)+\frac{C_{1-\beta} \Gamma(1+\beta) L b^{1-q+q \beta}(1+r)}{\beta \Gamma(1+q \beta)} \\
& +\frac{M}{\Gamma(q)}\left(\frac{1-p}{q-p} b\right)^{1-p}\|\phi\|_{L^{\frac{1}{p}}(J, X)}+\frac{M c b r}{\Gamma(q+1)} \\
\leq & r .
\end{aligned}
$$

Thus, $F$ maps $B_{r}$ into $B_{r}$.

Next, we will show that $F$ is continuous in $B_{r}$.

Let $\left\{x_{n}\right\} \in B_{r}$ with $x_{n} \rightarrow x$ in $B_{r}$, we have

$$
\begin{aligned}
t^{1-q} \| & \left(F x_{n}\right)(t)-(F x)(t) \| \\
\leq & t^{1-q}\left\|h\left(t, x_{n}(t)\right)-h(t, x(t))\right\| \\
& +t^{1-q}\left\|\int_{0}^{t}(t-s)^{q-1} A T_{q}(t-s)\left[h\left(s, x_{n}(s)\right)-h(s, x(s))\right] d s\right\| \\
& +t^{1-q}\left\|\int_{0}^{t}(t-s)^{q-1} T_{q}(t-s)\left[f\left(s, x_{n}(s)\right)-f(s, x(s))\right] d s\right\| \\
\leq & \left\|t^{1-q} A^{-\beta}\left[A^{\beta} h\left(t, x_{n}(t)\right)-A^{\beta} h(t, x(t))\right]\right\| \\
& +t^{1-q}\left\|\int_{0}^{t}(t-s)^{q-1} A^{1-\beta} T_{q}(t-s)\left[A^{\beta} h\left(s, x_{n}(s)\right)-A^{\beta} h(s, x(s))\right] d s\right\| \\
& +\frac{M t^{1-q}}{\Gamma(q)} \int_{0}^{t}(t-s)^{q-1}\left\|f\left(s, x_{n}(s)\right)-f(s, x(s))\right\| d s \\
\leq & b^{1-q} M_{0} L\left\|x_{n}-x\right\|_{C_{1-q}}+\frac{b^{1-q+q \beta} C_{1-\beta} \Gamma(1+\beta) L}{\beta \Gamma(1+q \beta)}\left\|x_{n}-x\right\|_{C_{1-q}} \\
& +\frac{M}{\Gamma(q)}\left(\frac{1-p}{q-p} b\right)^{1-p}\left\|G x_{n}-G x\right\|_{L^{\frac{1}{p}}(J, X)}
\end{aligned}
$$


which implies

$$
\left\|F x_{n}-F x\right\|_{C_{1-q}} \rightarrow 0 \quad \text { as } n \rightarrow \infty \text {. }
$$

This means that $F$ is continuous in $B_{r}$.

According to Lemma 3.1, $F_{1}\left(B_{r}\right)$ is relatively compact in $C_{1-q}(J, X)$, then $\alpha\left(F_{1}\left(B_{r}\right)\right)=0$.

For any $x_{1}, x_{2} \in B_{r}$, we have

$$
\begin{aligned}
t^{1-q} & \left\|\left(F_{2} x_{1}\right)(t)-\left(F_{2} x_{2}\right)(t)\right\| \\
\leq & t^{1-q}\left\|A^{-\beta}\left[A^{\beta} h\left(t, x_{1}(t)\right)-A^{\beta} h\left(t, x_{2}(t)\right)\right]\right\| \\
& +t^{1-q}\left\|\int_{0}^{t}(t-s)^{q-1} A^{1-\beta} T_{q}(t-s)\left[A^{\beta} h\left(s, x_{1}(s)\right)-A^{\beta} h\left(s, x_{2}(s)\right)\right] d s\right\| \\
\leq & b^{1-q} M_{0} L\left\|x_{1}-x_{2}\right\|_{C_{1-q}}+\frac{b^{1-q+q \beta} C_{1-\beta} \Gamma(1+\beta) L}{\beta \Gamma(1+q \beta)}\left\|x_{1}-x_{2}\right\|_{C_{1-q}},
\end{aligned}
$$

which implies that

$$
\left\|F_{2} x_{1}-F_{2} x_{2}\right\|_{C_{1-q}} \leq\left[b^{1-q} M_{0} L+\frac{b^{1-q(1-\beta)} C_{1-\beta} \Gamma(1+\beta) L}{\beta \Gamma(1+q \beta)}\right]\left\|x_{1}-x_{2}\right\|_{C_{1-q}} .
$$

Hence

$$
\alpha\left(F_{2}\left(B_{r}\right)\right) \leq\left[b^{1-q} M_{0} L+\frac{b^{1-q(1-\beta)} C_{1-\beta} \Gamma(1+\beta) L}{\beta \Gamma(1+q \beta)}\right] \alpha\left(B_{r}\right) .
$$

Therefore

$$
\begin{aligned}
\alpha\left(F\left(B_{r}\right)\right) & \leq \alpha\left(F_{1}\left(B_{r}\right)\right)+\alpha\left(F_{2}\left(B_{r}\right)\right) \\
& \leq\left[b^{1-q} M_{0} L+\frac{b^{1-q(1-\beta)} C_{1-\beta} \Gamma(1+\beta) L}{\beta \Gamma(1+q \beta)}\right] \alpha\left(B_{r}\right) .
\end{aligned}
$$

Noting that $M_{1}<1$, we find that the operator $F$ is an $\alpha$-contraction in $B_{r}$. It follows from Lemma 2.12 that $F$ has at least one fixed point in $B_{r}$. Then problem (1) has at least one mild solution in $B_{r}$. The proof is complete.

\subsection{The case that $T(t)$ is not compact}

For any $x \in B_{r}$, set $y(t)=t^{1-q} x(t)$, define $\mathcal{F}_{1}$ as follows:

$$
\begin{aligned}
\left(\mathcal{F}_{1} y\right)(t) & =t^{1-q}\left(F_{1} x\right)(t) \\
& =T_{q}(t) x_{0}+t^{1-q} \int_{0}^{t}(t-s)^{q-1} T_{q}(t-s) f(s, x(s)) d s, \quad t \in(0, b],
\end{aligned}
$$

and

$$
\left(\mathcal{F}_{1} y\right)(t)=\frac{x_{0}}{\Gamma(q)}, \quad t=0
$$

If $T(t)$ is noncompact, we will need the following assumptions. 
$\left(\mathrm{H}_{1}\right)^{\prime} T(t)(t>0)$ is continuous in the uniform operator topology for $t>0$.

$\left(\mathrm{H}_{5}\right)$ There exists a constant $\widehat{L}>0$ such that for any bounded $D \subset C_{1-q}(J, X)$,

$$
\alpha(f(t, D(t))) \leq \widehat{L} \alpha\left(t^{1-q} D(t)\right), \quad \text { for a.e. } t \in J
$$

Theorem 3.3 If assumptions $\left(\mathrm{H}_{1}\right)^{\prime},\left(\mathrm{H}_{2}\right)-\left(\mathrm{H}_{5}\right)$ are satisfied and $M_{1}<1$, then problem (1) has at least one mild solution.

Proof Define

$$
\widetilde{B}_{r}=\left\{h \in C(J, X):\|h\|_{C(J, X)} \leq r\right\} .
$$

For any $x \in B_{r}, y(t)=t^{1-q} x(t)$, it is easy to see that $y \in \widetilde{B}_{r}$. Let

$$
\Omega=\left\{\mathcal{F}_{1} y:\left(\mathcal{F}_{1} y\right)(t)=t^{1-q}\left(F_{1} x\right)(t), x \in B_{r}\right\}
$$

According to $\left(\mathrm{H}_{1}\right)^{\prime}$, Step 1 and Step 2 of Lemma 3.1, we find that $\mathcal{F}_{1}\left(\widetilde{B}_{r}\right) \subseteq \widetilde{B}_{r}$ and $\mathcal{F}_{1}\left(\widetilde{B}_{r}\right)$ is equicontinuous. Let $B=\overline{c o} \mathcal{F}_{1}\left(\widetilde{B}_{r}\right)$ then $B \subseteq \widetilde{B}_{r}$ is a bounded, closed, and convex set. According to Proposition 2.7, $B$ is also equicontinuous. Since

$$
B=\overline{c o} \mathcal{F}_{1}\left(\widetilde{B}_{r}\right) \subseteq \widetilde{B}_{r}
$$

we have $\mathcal{F}_{1}(B) \subseteq B$. Using a similar method as in the proof of Theorem 3.2, we can easily see that $\mathcal{F}_{1}: B \rightarrow B$ is continuous. For any bounded subset $B_{0} \subset B$, set

$$
\mathcal{F}_{1}^{1}\left(B_{0}\right)=\mathcal{F}_{1}\left(B_{0}\right), \quad \mathcal{F}_{1}^{n}\left(B_{0}\right)=\mathcal{F}_{1}\left(\overline{c o}\left(\mathcal{F}^{n-1}\left(B_{0}\right)\right)\right), \quad n=2,3, \ldots
$$

We know from Propositions 2.8-2.10, for any $\varepsilon>0$, that there is a sequence $\left\{u_{n}\right\}_{n=1}^{\infty} \subset B_{0}$, such that

$$
\begin{aligned}
\alpha\left(\left(\mathcal{F}_{1}^{1} B_{0}\right)(t)\right) & \leq 2 \alpha\left(\left\{\left(\mathcal{F}_{1} u_{n}\right)(t)\right\}_{n=1}^{\infty}\right)+\varepsilon \\
& =2 \alpha\left(T_{q}(t) x_{0}+t^{1-q} \int_{0}^{t}(t-s)^{q-1} T_{q}(t-s) f\left(s,\left\{x_{n}(s)\right\}_{n=1}^{\infty}\right) d s\right)+\varepsilon \\
& \leq \frac{4 M b^{1-q}}{\Gamma(q)} \int_{0}^{t}(t-s)^{q-1} \alpha\left(f\left(s,\left\{x_{n}(s)\right\}_{n=1}^{\infty}\right)\right) d s+\varepsilon \\
& \leq \frac{4 M b^{1-q} \widehat{L}}{\Gamma(q)} \int_{0}^{t}(t-s)^{q-1} \alpha\left(s^{1-q}\left\{x_{n}(s)\right\}_{n=1}^{\infty}\right) d s+\varepsilon \\
& =\frac{4 M b^{1-q} \widehat{L}}{\Gamma(q)} \int_{0}^{t}(t-s)^{q-1} \alpha\left(\left\{u_{n}(s)\right\}_{n=1}^{\infty}\right) d s+\varepsilon \\
& \leq \frac{4 M \widehat{L} b^{1-q} \alpha\left(B_{0}\right) t^{q}}{\Gamma(q+1)}+\varepsilon .
\end{aligned}
$$

Since $\varepsilon>0$ is arbitrary, we have

$$
\alpha\left(\left(\mathcal{F}_{1}^{1} B_{0}\right)(t)\right) \leq \frac{4 M \widehat{L} b^{1-q} \alpha\left(B_{0}\right) t^{q}}{\Gamma(q+1)} .
$$


For any $\varepsilon>0$, there is a sequence $\left\{y_{n}\right\}_{n=1}^{\infty} \subset \overline{c o}\left(F^{1}\left(B_{0}\right)\right)$, such that

$$
\begin{aligned}
\alpha\left(\left(\mathcal{F}_{1}^{2} B_{0}\right)(t)\right) & \leq 2 \alpha\left(\left\{\left(\mathcal{F}_{1} y_{n}\right)(t)\right\}_{n=1}^{\infty}\right)+\varepsilon \\
& =2 \alpha\left(T_{q}(t) x_{0}+t^{1-q} \int_{0}^{t}(t-s)^{q-1} T_{q}(t-s) f\left(s,\left\{x_{n}(s)\right\}_{n=1}^{\infty}\right) d s\right)+\varepsilon \\
& \leq \frac{4 M b^{1-q}}{\Gamma(q)} \int_{0}^{t}(t-s)^{q-1} \alpha\left(f\left(s,\left\{x_{n}(s)\right\}_{n=1}^{\infty}\right)\right) d s+\varepsilon \\
& \leq \frac{4 M b^{1-q} \widehat{L}}{\Gamma(q)} \int_{0}^{t}(t-s)^{q-1} \alpha\left(s^{1-q}\left\{x_{n}(s)\right\}_{n=1}^{\infty}\right) d s+\varepsilon \\
& =\frac{4 M b^{1-q} \widehat{L}}{\Gamma(q)} \int_{0}^{t}(t-s)^{q-1} \alpha\left(\left\{y_{n}(s)\right\}_{n=1}^{\infty}\right) d s+\varepsilon \\
& \leq \frac{4 M b^{1-q} \widehat{L}}{\Gamma(q)} \int_{0}^{t}(t-s)^{q-1} \alpha\left(\left(\mathcal{F}_{1}^{1} B_{0}\right)(s)\right) d s+\varepsilon \\
& \leq \frac{4 M \widehat{L} b^{1-q}}{\Gamma(q)} \int_{0}^{t}(t-s)^{q-1} \times \frac{4 M L b^{1-q} s^{q}}{\Gamma(q+1)} \alpha\left(B_{0}\right) d s+\varepsilon \\
& \leq \frac{\left(4 M \widehat{L} b^{1-q} t^{q}\right)^{2}}{\Gamma(2 q+1)} \alpha\left(B_{0}\right)+\varepsilon
\end{aligned}
$$

Since $\varepsilon>0$ is arbitrary, we have

$$
\alpha\left(\left(\mathcal{F}_{1}^{2} B_{0}\right)(t)\right) \leq \frac{\left(4 M \widehat{L} b^{1-q} t^{q}\right)^{2}}{\Gamma(2 q+1)} \alpha\left(B_{0}\right) .
$$

It can be shown by mathematical induction that

$$
\begin{aligned}
\alpha\left(\left(\mathcal{F}_{1}^{n} B_{0}\right)(t)\right) & \leq \frac{\left(4 M \widehat{L} b^{1-q} t^{q}\right)^{n}}{\Gamma(n q+1)} \alpha\left(B_{0}\right) \\
& \leq \frac{(4 M \widehat{L} b)^{n}}{\Gamma(n q+1)} \alpha\left(B_{0}\right)
\end{aligned}
$$

By using the well-known Stirling formula, we get

$$
\Gamma(1+n q)=\sqrt{2 \pi n q}\left(\frac{n q}{e}\right)^{n q} e^{\frac{\theta}{12 n q}}, \quad 0<\theta<1 .
$$

Then

$$
\lim _{n \rightarrow \infty} \frac{(4 M \widehat{L} b)^{n}}{\Gamma(n q+1)}=\lim _{n \rightarrow \infty} \frac{(4 M \widehat{L} b)^{n}}{\sqrt{2 \pi n q}\left(\frac{n q}{e}\right)^{n q} e^{\frac{\theta}{12 n q}}}=0 .
$$

Then there exists a $\gamma \in(0,1)$ such that

$$
\alpha\left(\left(\mathcal{F}_{1}^{n} B_{0}\right)(t)\right) \leq \gamma \alpha\left(B_{0}\right)
$$

It can be shown that $\mathcal{F}_{1}^{n} B_{0} \subseteq B$. Since $B$ is bounded and equicontinuous, $\mathcal{F}_{1}^{n} B_{0}$ is also bounded and equicontinuous. It follows from Proposition 2.8 that

$$
\alpha\left(\mathcal{F}_{1}^{n} B_{0}\right)=\max _{t \in J} \alpha\left(\left(\mathcal{F}_{1}^{n} B_{0}\right)(t)\right) \leq \gamma \alpha\left(B_{0}\right)
$$


Then, by Lemma 2.11, there exists a $\bar{D} \subset B \subset \widetilde{B_{r}}$ such that

$$
\alpha\left(\mathcal{F}_{1}(\bar{D})\right)=0
$$

Let

$$
Q=\left\{x \in C_{1-q}(J, X): x(t)=t^{q-1} y(t), y \in \bar{D}\right\} .
$$

For any $x \in Q$, we have $\|x\|_{C_{1-q}}=\|y\|_{C(J, X)} \leq r$, which means that $Q \subseteq B_{r}$. Since $\mathcal{F}_{1}(\bar{D})$ is relatively compact, we can easily prove that $F_{1}(Q)$ is relatively compact, that is

$$
\alpha\left(F_{1}(Q)\right)=0
$$

On the other hand, for any $x_{1}, x_{2} \in Q$, we have

$$
\begin{aligned}
t^{1-q} & \left\|\left(F_{2} x_{1}\right)(t)-\left(F_{2} x_{2}\right)(t)\right\| \\
\leq & t^{1-q}\left\|A^{-\beta}\left[A^{\beta} h\left(t, x_{1}(t)\right)-A^{\beta} h\left(t, x_{2}(t)\right)\right]\right\| \\
& \quad+t^{1-q}\left\|\int_{0}^{t}(t-s)^{q-1} A^{1-\beta} T_{q}(t-s)\left[A^{\beta} h\left(s, x_{1}(s)\right)-A^{\beta} h\left(s, x_{2}(s)\right)\right] d s\right\| \\
\leq & b^{1-q} M_{0} L\left\|x_{1}-x_{2}\right\|_{C_{1-q}}+\frac{b^{1-q(1-\beta)} C_{1-\beta} \Gamma(1+\beta) L}{\beta \Gamma(1+q \beta)}\left\|x_{1}-x_{2}\right\|_{C_{1-q}},
\end{aligned}
$$

which implies that

$$
\left\|F_{2} x_{1}-F_{2} x_{2}\right\|_{C_{1-q}} \leq\left[b^{1-q} M_{0} L+\frac{b^{1-q(1-\beta)} C_{1-\beta} \Gamma(1+\beta) L}{\beta \Gamma(1+q \beta)}\right]\left\|x_{1}-x_{2}\right\|_{C_{1-q}} .
$$

Hence

$$
\alpha\left(F_{2}(Q)\right) \leq\left[b^{1-q} M_{0} L+\frac{b^{1-q(1-\beta)} C_{1-\beta} \Gamma(1+\beta) L}{\beta \Gamma(1+q \beta)}\right] \alpha(Q) .
$$

Therefore

$$
\alpha(F(Q)) \leq \alpha\left(F_{1}(Q)\right)+\alpha\left(F_{2}(Q)\right) \leq\left[b^{1-q} M_{0} L+\frac{b^{1-q(1-\beta)} C_{1-\beta} \Gamma(1+\beta) L}{\beta \Gamma(1+q \beta)}\right] \alpha(Q) .
$$

Noting that $M_{1}<1$, thus the operator $F$ is an $\alpha$-contraction in $Q$. It follows from Lemma 2.12 that $F$ has at least one fixed point in $Q$. Then problem (1) has at least one mild solution in $Q$. The proof is complete.

\section{An example}

Let $X=L^{2}([0, \pi], \mathbb{R})$. As an application of our results, consider the following fractional partial differential equations:

$$
\left\{\begin{array}{c}
{ }^{L} D_{0+}^{\frac{1}{2}}\left[z(t, x)-\sin \left(\int_{0}^{\pi} b(t, y, x)\left[z(t, y)+\frac{\partial}{\partial y} z(t, y)\right] d y\right)\right] \\
=\frac{\partial^{2}}{\partial x^{2}} z(t, x)+f(t, z(t, x)), \quad t \in(0, b], x \in[0, \pi], \\
z(t, 0)=z(t, \pi)=0, \quad t \in(0, b] \\
\left.I_{0^{+}}^{\frac{1}{2}} z(t, x)\right|_{t=0}=z_{0}(x), \quad x \in[0, \pi],
\end{array}\right.
$$


where ${ }^{L} D_{0+}^{\frac{1}{2}}$ is the Riemann-Liouville fractional derivative of order $\frac{1}{2}, b>0, I_{0^{+}}^{\frac{1}{2}}$ is a Riemann-Liouville integral of order $\frac{1}{2}$. Define the operator $A$ by $A z=-z^{\prime \prime}$, with the domain

$$
D(A)=\left\{z(\cdot) \in X: z, z^{\prime} \text { are absolutely continuous, } z^{\prime \prime} \in X, z(t, 0)=z(t, \pi)=0\right\} .
$$

Then $-A$ generates a strongly continuous semigroup $\{T(t)\}_{t \geq 0}$ which is compact, analytic. Moreover, $A$ can be written as

$$
A z=\sum_{n=1}^{\infty} n^{2}<z, \quad e_{n}>e_{n}, z \in D(A)
$$

where $e_{n}(x)=\sqrt{\frac{2}{\pi}} \sin n x, 0 \leq x \leq \pi, n=1,2, \ldots$ is an orthonormal basis of $X$. We have

$$
T(t) z=\sum_{n=1}^{\infty} e^{-n^{2} t}<z, \quad e_{n}>e_{n}, z \in X, \quad \text { and } \quad\|T(t)\| \leq e^{-t} \leq 1=M, \quad t \geq 0 .
$$

For each $z \in X$,

$$
A^{-\frac{1}{2}} z=\sum_{n=1}^{\infty} \frac{1}{n}<z, \quad e_{n}>e_{n} ;
$$

in particular, $\left\|A^{-\frac{1}{2}}\right\|=1$.

The operator $A^{\frac{1}{2}}$ is given by

$$
A^{\frac{1}{2}} z=\sum_{n=1}^{\infty} n<z, \quad e_{n}>e_{n}
$$

on the space

$$
D\left(A^{\frac{1}{2}}\right)=\left\{z(\cdot) \in X, \sum_{n=1}^{\infty} n<z, e_{n}>e_{n} \in X\right\} .
$$

Choose $q=\beta=\frac{1}{2}$, define

$$
\begin{aligned}
& z(t, x)=z(t)(x), \quad f(t, z(t, x))=f(t, z(t))(x), \\
& h(t, z)(x)=\sin \left(\int_{0}^{\pi} b(t, y, x)\left[z(t, y)+\frac{\partial}{\partial y} z(t, y)\right] d y\right),
\end{aligned}
$$

then system (5) can be written in the abstract form given by (1). Assume that $\left(\mathrm{H}_{2}\right)-\left(\mathrm{H}_{4}\right)$ are satisfied and $M_{1}<1$; then by Theorem 3.2, system (5) has at least one mild solution.

\section{Conclusions}

In this manuscript, by using the fractional power of operators and the theory of a measure of noncompactness, the existence of fractional neutral evolution equations with a Riemann-Liouville fractional derivative were investigated. We give an example to illustrate the applications of the abstract results. 


\section{Competing interests}

The authors declare that they have no competing interests.

\section{Authors' contributions}

All authors contributed equally and significantly in writing this paper. All authors read and approved the final manuscript.

\section{Acknowledgements}

The authors would like to express their gratitude to the editor and referees for their careful reading of the manuscript and a number of excellent criticisms and suggestions. The work is financially supported by the NNSF of China Grant Nos.

11271087,61263006 and Innovation Project of Guangxi University for Nationalities (gxun-chx2012101, gxun-ch×2013082).

\section{Received: 30 October 2013 Accepted: 28 February 2014 Published: 12 Mar 2014}

\section{References}

1. Samko, SG, Kilbas, AA, Marichev, Ol: Fractional Integral and Derivatives, Theory and Applications. Gordon \& Breach, Yverdon (1993)

2. Kilbas, AA, Srivastava, HM, Trujillo, JJ: Theory and Applications of Fractional Differential Equations. North-Holland Mathematics Studies. Elsevier, Amsterdam (2006)

3. Miller, KS, Ross, B: An Introduction to the Fractional Calculus and Differential Equations. Wiley, New York (1993)

4. Podlubny, I: Fractional Differential Equations. Academic Press, San Diego (1999)

5. Heymans, N, Podlubny, I: Physical interpretation of initial conditions for fractional differential equations with Riemann-Liouville fractional derivatives. Rheol. Acta 45, 765-771 (2006)

6. Li, F, Liang, J, Xu, HK: Existence of mild solutions for fractional integrodifferential equations of Sobolev type with nonlocal conditions. J. Math. Anal. Appl. 391, 510-525 (2012)

7. Liu, ZH, Sun, JH, Szanto, I: Monotone iterative technique for Riemann-Liouville fractional integro-differential equations with advanced arguments. Results Math. 63, 1277-1287 (2013)

8. $\mathrm{Liu}, \mathrm{ZH}, \mathrm{Li}, \mathrm{XW}$ : Existence and uniqueness of solutions for the nonlinear impulsive fractional differential equations. Commun. Nonlinear Sci. Numer. Simul. 18, 1362-1373 (2013)

9. Liu, ZH, Li, XW: On the controllability of impulsive fractional evolution inclusions in Banach spaces. J. Optim. Theory Appl. 156, 167-182 (2013)

10. Liu, ZH, Wang, R: A note on fractional equations of Volterra type with nonlocal boundary condition. Abstr. Appl. Anal. 2013, Article ID 432941 (2013)

11. Wang, JR, Zhou, Y, Fečkan, M: Abstract Cauchy problem for fractional differential equations. Nonlinear Dyn. 71, 685-700 (2013)

12. Zhou, Y, Jiao, F: Existence of mild solutions for fractional neutral evolution equations. Comput. Math. Appl. 59 1063-1077 (2010)

13. Zhou, Y, Jiao, F, Li, J: Existence and uniqueness for fractional neutral differential equations with infinite delay. Nonlinear Anal. 71, 3249-3256 (2009)

14. Zhou, $Y$, Jiao, F, Li, J: Existence and uniqueness for $p$-type fractional neutral differential equations. Nonlinear Anal. 71, 2724-2733 (2009)

15. Liu, ZH, Li, XW Sun, JH: Controllability of nonlinear fractional impulsive evolution systems. J. Integral Equ. Appl. 25 395-405 (2013)

16. Zhou, Y, Zhang, L, Shen, XH: Existence of mild solutions for fractional evolution equations. J. Integral Equ. Appl. 25, 455-600 (2013)

17. Agarwal, RP, Belmekki, M, Benchohra, M: A survey on semilinear differential equations and inclusions involving Riemann-Liouville fractional derivative. Adv. Differ. Equ. 2009, Article ID 981728 (2009)

18. Agarwal, RP, de Andrade, B, Cuevas, C: On type of periodicity and ergodicity to a class of fractional order differential equations. Adv. Differ. Equ. 2010, Article ID 179750 (2010)

19. dos Santos, JPC, Mallika Arjunan, M, Cuevas, C: Existence results for a fractional neutral integro-differential equations with state dependent delay. Comput. Math. Appl. 62, 1275-1283 (2011)

20. Goldstein, JA: Semigroups of Linear Operators and Applications. Oxford University Press, New York (1985)

21. Pazy, A: Semigroups of Linear Operators and Applications to Partial Differential Equations. Springer, New York (1983)

22. Akhmerov, RR, Kamenskij, MI, Potapov, AS, Rodkina, AE, Sadovskij, BN: Measures of Noncompactness and Condensing Operators. Nauka, Novosibirsk (1986) (in Russian). Engl. transl.: Birkhäuser, Basel (1992)

23. Kamenskii, M, Obukhovskii, V, Zecca, P: Condensing Multi-Valued Maps and Semilinear Differential Inclusions in Banach Spaces. de Gruyter, Berlin (2001)

24. Banaš, J, Goebel, K: Measure of Noncompactness in Banach Space. Dekker, New York (1980)

25. Deimling, K, Goebel, K: Nonlinear Functional Analysis. Springer, Berlin (1985)

26. Lakshmikantham, V, Leela, S: Nonlinear Differential Equations in Abstract Spaces. Pergamon, New York (1969)

27. Guo, DJ, Lakshmikantham, V, Liu, XZ: Nonlinear Integral Equations in Abstract Spaces. Kluwer Academic, Dordrecht (1996)

28. Bothe, D: Multivalued perturbation of $m$-accretive differential inclusions. Isr. J. Math. 108, 109-138 (1998)

29. Mönch, $\mathrm{H}$ : Boundary value problems for nonlinear ordinary differential equations of second order in Banach space. Nonlinear Anal. TMA 4, 985-999 (1980)

30. Liu, L, Guo, F, Wu, C, Wu, Y: Existence theorems of global solutions for nonlinear Volterra type integral equations in Banach spaces. J. Math. Anal. Appl. 309, 638-649 (2005)

10.1186/1687-1847-2014-83

Cite this article as: Liu and Lv: Existence results for Riemann-Liouville fractional neutral evolution equations. Advances in Difference Equations 2014, 2014:83 